\title{
Transcriptional profiling by RNA sequencing of black pepper (Piper nigrum L.) roots infected by Fusarium solani f. sp. piperis
}

\author{
Edith C. O. Moreira ${ }^{1}$ Daniel G. Pinheiro ${ }^{2,3} \cdot$ Sheila M. C. Gordo ${ }^{1}$. \\ Simone M. Rodrigues ${ }^{4}$ Elaine Pessoa ${ }^{5,7} \cdot$ Hubert Schaller $^{6,7} \cdot$ Oriel F. de Lemos $^{4}$. \\ Artur Silva $^{5} \cdot$ Horacio Schneider $^{1} \cdot$ Wilson A. Silva Jr. $^{2} \cdot$ Iracilda Sampaio $^{1}$ • \\ Sylvain Darnet ${ }^{5,7}$ (i)
}

Received: 12 April 2017/Revised: 9 July 2017 / Accepted: 12 September 2017 /Published online: 21 September 2017

(C) Franciszek Górski Institute of Plant Physiology, Polish Academy of Sciences, Kraków 2017

\begin{abstract}
Black pepper (Piper nigrum L.) is the most traded spice worldwide and therefore has great economic value. One of the major limitations of its production is yield losses in fields due to root rot, a disease caused by Fusarium solani f. sp. piperis. This soil-borne pathogen disseminates rapidly in tropical countries. Biotechnological breeding is often presented as an efficient tool to produce resistant pepper cultivars. An RNA-sequencing experiment was used to take a snapshot of the root transcriptome 60 days after infection by the pathogen. The mapping of 67 million SOLiD single-end reads to the Fusarium genome identified many fungal transcripts coding constitutive proteins and two proteins involved in virulence and conidial formation. The annotation of black pepper transcripts obtained by de novo assembly predicted three proteins restricted to this species. While these transcripts were upregulated in infected roots, the corresponding predicted
\end{abstract}

Communicated by E. Kuzniak-Gebarowska.

Electronic supplementary material The online version of this article (doi:10.1007/s11738-017-2538-y) contains supplementary material, which is available to authorized users.

Sylvain Darnet

shd@laposte.net

1 Genetics and Molecular Biology Laboratory, Coastal Studies Institute, Bragança Campus, Universidade Federal do Pará, Bragança, Pará, Brazil

2 Department of Genetics, Faculty of Medicine of Ribeirão Preto, University of São Paulo, Ribeirão Preto, São Paulo, Brazil

3 School of Agricultural and Veterinary Sciences, São Paulo State University (Unesp), Jaboticabal, São Paulo, SP, Brazil

4 Brazilian Agricultural Research Corporation - EMBRAPA Amazônia Oriental, Belém, Pará, Brazil proteins had no hit in databases. A global analysis of differentially expressed black pepper genes, using terms of gene ontology, has demonstrated an enrichment of genes involved in proteolysis, plastid degradation, cell-wall remodeling and secondary metabolism, consistent with toxicity and necrotrophic fungal interactions that force plants to collaborate at the metabolic level. Detailed descriptions of up- or downregulated genes associated with plant defense suggested several genes implied in the biosynthesis of isoprenoids, especially for an upregulated terpene synthase, a putative geranylgeranyl diphosphate synthase, and a downregulated brassinosteroid signaling component $(B A K 1)$. These results provide new clues for investigating further target genes and biotechnological approaches for breeding black pepper resistance to Fusarium.

Keywords Differential expression - Root rot · Piper nigrum $\cdot$ RNA-seq $\cdot$ Fusarium

5 Instituto de Ciências Biológicas, Universidade Federal do Pará, Belém, PA, Brazil

6 Plant Isoprenoid Biology (PIB) Team, Institut de Biologie Moléculaire des Plantes du CNRS, Université de Strasbourg, Strasbourg, France

7 Laboratoire International Associé PALMHEAT CNRS/ UFPA, Direction Europe de la Recherche et Coopération Internationale (DERCI)- Campus, Gérard-Mégie 3, rue Michel-Ange, 75794 Paris Cedex 16, France 


\section{Introduction}

The genus Piper is highly diverse, containing about 2000 species, and is distributed at tropical latitudes. Many of these species are of biotechnological interest due to their high contents of active compounds such as the lysinederived piperidine, quinolizidine, indolizidine and lycopodium alkaloids (Jaramillo and Manos 2001; Menezes et al. 2009). Black pepper (Piper nigrum L.) is the most traded spice in the world, widely used in cooking, food preservation and perfume production. Some black pepper chemicals also have medicinal properties, such as antioxidant, anti-inflammatory and anticancer activities (Liu et al. 2010; Meghwal and Goswami 2013; Nishimura et al. 2011). The global production of black pepper from tropical countries is about 334,000 tonnes, totaling 1.8 billion US dollars. Up to $83 \%$ of this production is imported to Europe and North America (FAO 2011). This culture consequently has great economic and social importance in developing countries.

Phytopathological problems such as foot rot and root rot caused by fungi from the genera Phytophthora and Fusarium, respectively, represent a major threat to global Piper production (Duarte and Albuquerque 2001; Joy et al. 2007). Fusarium solani f. sp. piperis (Fsp) and its teleomorphic form known as Nectria haematococca f. sp. piperis, is a biotrophic soil fungus belonging to the class Ascomycetes that is endemic to the Brazilian Amazon region. The temperature and humidity in this region are constantly high and so offer optimal conditions for pathogen sporulation, which increases pathogen dissemination. Many black pepper plantations have thus been contaminated, affecting yield production and drupe quality (Albuquerque 1961; Albuquerque et al. 2001; Hamada et al. 1988). The pathogenic infection impairs the conducting vessels by increasing mycelial mass, leading to decreases in the supply of water and nutrients to the plant (Albuquerque et al. 2001; Fukutomi and Hirakata 1981). The physiology of the infection and disease is well known, but their underlying molecular mechanisms are poorly understood.

Evidence suggests that initial infections by root pathogens are generally established in the root elongation zone, as demonstrated for Pisum sativum (Cannesan et al. 2011; Gunawardena et al. 2005). The molecular plant-host interaction, however, is better known for foliar than root pathogens, which are poorly characterized and understood (Balmer and Mauch-Mani 2013; Okubara and Paulitz 2005). The elucidation at the molecular level of the defensive responses involved in the radicular pathosystem can consequently assist our understanding of these interactions and help us to design efficient biotechnological approaches for plant breeding. Molecular interactions that characterize the interaction of Fusarium with its host plants have been successfully identified using transcriptomic profiles obtained by RNA sequencing (RNA-seq), in both model fungus/plant systems such as Fusarium oxysporum/ Arabidopsis thaliana (L.) Heynh and in plants of agronomic interest (Berrocal-Lobo and Molina 2008). A previous experiment using black pepper suppression subtractive hybridization libraries has provided a partial view of the genes expressed in Fsp-plant interactions (de Souza et al. 2011).

We report an exhaustive analysis of the entire transcriptome of black pepper roots inoculated by Fsp, using RNA-seq to identify differentially expressed genes and to infer the global molecular mechanisms involved in plant defense against pathogenic fungi.

\section{Materials and methods}

\section{Biological material}

This study was approved by IBAMA under license number 02001.006478/2010-49 and special authorization 02/2008. Black pepper plants (cv. Bragantina) were inoculated with an isolate of Fusarium solani f. sp. piperis, provided by the Collection of Fungi and Phytopathogens of Amazon Biome at Embrapa Amazônia Oriental (de Castro et al. 2016). This isolate originated from black pepper plant roots with symptoms of fusariosis, collected in the Municipality of Baiao, Para, Brazil (de Castro et al. 2016). Two groups of thirty-five plants each were separately cultivated in a greenhouse: one group of control plants was inoculated with water, and the other group was inoculated with Fsp. The method of inoculation was based on the protocol described by Albuquerque et al. (2001). The pathogen was cultivated at $25{ }^{\circ} \mathrm{C}$ under a 12 -h light/dark cycle on a water-agar culture medium containing potato dextrose. Disks of conidia and mycelia $0.7 \mathrm{~cm}$ in diameter were collected from these cultures and prepared as soil inocula. The black pepper plants were potted in vermiculite containing the Fusarium inoculum. The first symptoms of root rot were visible 60 days after inoculation, and plants from the above-mentioned two groups were then collected (Albuquerque 1961; de Castro et al. 2016).

\section{cDNA preparation and SOLiD sequencing}

Ten plants were randomly selected in each of the two groups of 35 plants inoculated with water or Fsp. Stem tissues and root-zone elongation tissues were sampled form 
these pools of ten plants (Online resource 1). Total RNA was extracted from about $35 \mathrm{mg}$ of fresh tissue. A noninoculated root library (NIRL) and an infected root library (IRL) were prepared from these RNAs and sequenced on a SOLiD Next-Generation Sequencer (version 3.0), as described by Gordo et al. (2012) (Thermo Fisher Scientific, Waltham, USA). Library preparation and sequencing were not replicated for this experiment. The NIRL was previously published and registered as SRR1164727 in the NCBI-SRA database (Gordo et al. 2012). The accession number for the IRL reported in this study is SRR654187.

\section{Bioinformatic analysis}

A transcript sequence assembly (TSA) was obtained using the hybrid color-space read-additive method (Viegas et al. 2015), with a further step for UniVec removal of contaminated sequences (https://www.ncbi.nlm.nih.gov/tools/ vecscreen/univec/), and deposited under accession GFIY00000000 in the NCBI database. IRL and NIRL read data sets were mapped on TSA contigs for transcript quantification using CLC Genomics Workbench (CLCg) with default parameters (https://www.qiagenbioinfor matics.com). The transcripts were functionally annotated using Blast2GO (Conesa and Gotz 2008), with an initial comparison of the TSA data set with the Swiss-Prot/Uniprot database (http://www.uniprot.org) using BLASTX with an $E$-value of $1 \mathrm{e}-3$ (https://blast.ncbi.nlm.nih.gov/ Blast.cgi). The nucleotides of orphan gene sequences were compared using SRA-BLAST and NGS read data sets of leaf and drupe libraries, sequenced by HiSeq and MiSeq Illumina platforms and registered under NCBI accession SRX119532，SRX1716592，SRX2855187，SRX853366, SRX856632, SRX856639 and SRX890122 (https://blast. ncbi.nlm.nih.gov/Blast.cgi?PROGRAM=blastn\&BLAST_ PROGRAMS=megaBlast\&PAGE_TYPE=BlastSearch\& BLAST_SPEC $=$ SRA\&SHOW_DEFAULTS $=$ on). For further characterization and modelling of molecular responses, black pepper transcripts were clustered by $A$. thaliana orthologs using the Araport 11 protein data set (https://www.araport.org) and BLASTX with an $E$-value of 1e-3 as described for de novo transcriptomes in non-model species (Nogueira et al. 2016). Transcript expression was calculated for each A. thaliana homologous-gene cluster (AHGC) by summing the read counts for each black pepper transcript in the cluster. Identification of differentially expressed genes was based on Kal's Z-test in CLCg. The enrichment of terms of gene ontology (GO) between conditions was globally analyzed using the parametric analysis of gene set enrichment (PAGE) tool of the agriGO portal (Du et al. 2010). The AHGC expression data set was imported into MapMan for visualizing and interpreting the global molecular responses (Jung and An 2013). For the digital expression of Fsp genes, IRL and NIRL read data sets were mapped with the CLCg mapper on the $N$. haematococca mpVI 77-13-4 genome (https://www.ncbi. nlm.nih.gov/assembly/GCF_000151355.1/), with a length fraction of $95 \%$ and a similarity fraction of $98 \%$ to ensure high-specificity mapping. Statistical analysis was performed using CLCg and Kal's Z-test.

\section{Results and discussion}

\section{Library sequencing}

We generated an IRL from RNAs extracted from a pool of ten plants to minimize individual variation. All plants displayed symptoms of Fusarium root rot such as blackened aspect of roots and collars and yellowish leaves (Albuquerque 1961) (Online resource 1). The blackened aspect of infected roots was most probably due to the production of toxins by the Fsp isolate (de Castro et al. 2016) that caused the symptoms of the disease on aboveground plant tissues, such as yellowish leaves and dried branches, and ultimately plant death (Albuquerque 1961; Albuquerque et al. 2001; de Castro et al. 2016; Duarte and Albuquerque 2001). All non-inoculated plants that were sampled to generate an NIRL had very clear roots (Online resource 1). NIRL and IRL were single-end sequenced using the SOLiD version 3 platform, and the raw sequence data sets contained about 72 million $(3.57 \mathrm{Gbp})$ and 54 million ( $2.74 \mathrm{Gbp})$ reads, respectively.

\section{Analysis of digital gene expression (DGE) of Fsp reads}

Reads from Fsp were first identified by mapping the read data sets from NIRL and IRL on the $N$. haematococca genome, the teleomorph of $F$. solani. The mapping found only 180 mapped reads for NIRL and 31,186 reads for IRL. Transcripts should be considered non-expressed and under the limit of detection if they have $<100$ mapped reads or $<1$ transcript per million (TPM) reads. For NIRL, no genes were detected with values $>100$ mapped reads. For IRL, $60 \%$ of the reads (19,067 reads) were mapped in exons and $41.5 \%$ in introns, consistent with the use for library preparation of total RNAs depleted in rRNAs and containing ncRNAs. The low level of detected reads from pathogenic fungi, in this case $0.13 \%$ of the total reads, was previously reported for another pathosystem (Petre et al. 2012). The list of genes expressed with $>100$ reads is presented in Online Resource 2. Many of the detected genes were constitutive and should be considered fungal house-keeping genes, such as calmodulin, actin and tubulin, or genes involved in metabolism. Only a few candidate 
genes were associated with plant-host interactions, such as the interferon-regulated resistance GTP-binding protein already isolated during the biosynthesis of zearalenone in the genus Fusarium (Lysøe et al. 2008). A molecular chaperone encoded by $H T P G$, which plays a crucial role in the vegetative growth, reproduction and virulence of $F$. graminearum (Bui et al. 2016), was expressed. Interestingly, the two most abundant transcripts were not well annotated, one without homology in the non-redundant (nr) NCBI database and the other with a low similarity to a histone-lysine N-methyltransferase and to the H3 lysine- 9 specific dim-5 gene from Neonectria ditissima, a protein regulator of the circadian cycle and conidial formation (Ruesch et al. 2014). Only highly abundant genes were detected. These genes were annotated as constitutive genes, one uncharacterized gene and three putative central regulators of Fusarium reproduction and virulence.

\section{DGE analysis of black pepper transcripts in roots infected by Fsp}

\section{Transcriptome assembly and annotation}

The NIRL and IRL reads were mapped on the TSA data set as described by Viegas et al. (2015) for evaluating the abundance of black pepper transcripts. The TSA data set was obtained using a hybrid method to assemble the SOLiD single-end reads with Illumina NGS paired-end reads obtained from the same cultivar of black pepper by Joy et al. (2013). This reference transcriptome contained about $65.4 \mathrm{Mbp}$, with 59,863 predicted transcripts, an N50 of $1762 \mathrm{bp}, 27,747$ sequences longer than $1000 \mathrm{bp}$ and a CEGMA score for complete genes of about $98 \%$, indicating that the assembly was successful (Viegas et al. 2015). The annotation was performed by comparison with the Swiss-Prot database: 17,807 transcripts were fully annotated and 12,185 had homologies lower than 1e-100 with an orthologous sequence. All transcript annotations and information retrieved with Blast2Go as GO terms are listed in Online Resource 3. The total mapped reads were $15,484,438$ for NIRL and 12,524,954 for IRL, representing 82.5 and $52.1 \%$ of the preprocessed read data sets, respectively. A statistical Z-test was performed between NIRL and IRL using the method described by Kal et al. (1999) for experiments with one sample per condition. The DGE analysis was interpreted in two steps: putative lineage-restricted genes upregulated in IRL and ncRNAs were first identified, then a global model of molecular responses was established based on the $A$. thaliana genome.
Prediction of orphan genes associated with Fsp infection

The orphan genes or genes restricted to $P$. nigrum, which were upregulated in IRL and associated with inoculation, were identified by filtering the transcripts based on the following criteria described by Nogueira et al. (2016): presence of a coding sequence, absence of homology in the UniProt/Viridiplantae or nr NCBI databases with $E$ values up to $1 \mathrm{e}-3$, more than two-fold increases between NIRL and IRL ( $\left.\mathrm{FC}_{\text {IRL/NIRL }}\right)$, transcripts longer than $500 \mathrm{bp}$, false discovery rate less than 0.05 , IRL read count over 100 and $P<0.001$. This selection identified three transcripts. Transcript sequences were compared to seven different NGS libraries of $P$. nigrum leaves and drupes available in NCBI database using SRA-BLASTN to confirm the presence of the transcripts and to reject the hypothesis of putative contaminating microbial sequences. More than 250 NGS reads were mapped on each transcript, and the top-100 BLAST subset had very high sequence identities, about 99-100\%, along the length of the three orphan gene transcripts, confirming the presence of these genes in the $P$. nigrum transcriptome (Online Resource 4). Two transcripts had full-length coding sequences (CDS): contig311778 with a predicted protein of 135 aa, and contig480387 with a predicted protein of 139 aa. A third transcript had a $5^{\prime}$ partial CDS that corresponded to contig524182 with a predicted protein of 122 aa. All predicted protein sequences had no hit in the nr NCBI database, providing an opportunity to discover new proteins involved in defensive mechanisms in basal angiosperms.

\section{$n c R N A s$ and ncRNA precursors}

IRL and NIRL were obtained from total RNAs that were depleted in rRNAs and consequently contained mRNAs but also ncRNA. Transcripts with no hit in the Viridiplantae/ Uniprot database were submitted as queries to the RFAM server for predicting possible ncRNAs and ncRNA precursors (Nawrocki et al. 2015). About 350 transcripts were annotated as ncRNAs, $87 \%$ of which were identified as rRNAs (LSU_rRNA, SSU_rRNAs and 5S) of archaebacteria, prokaryotes and eukaryotes (Online Resource 5). This result indicated that the depletion of rRNAs was not complete and the presence of microorganisms in the black pepper samples. These microorganisms most probably represented the background microbial population interacting with pepper plants grown in greenhouse conditions. Other classes of ncRNAs precursors were identified as miRNAs and snoRNAs. Among four microRNA 


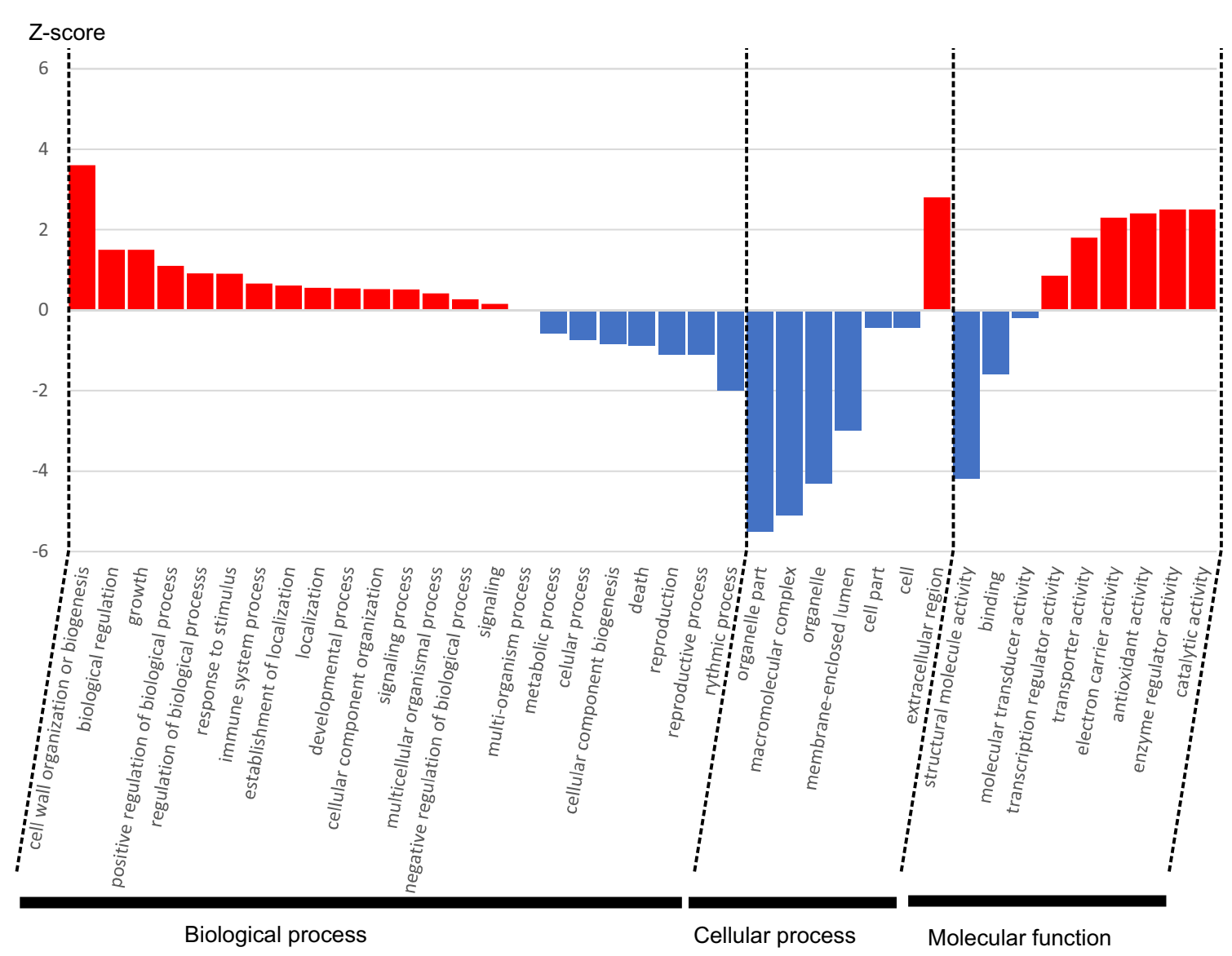

Fig. 1 Global enrichment and decrease in GO terms comparing black pepper transcripts, clustered by Arabidopsis thaliana orthologs, differentially expressed between roots uninfected and infected by

precursors, miR-171 and miR-166 had already been described in black pepper (Asha et al. 2012). All these miRNAs were expressed in root tissue, which did not substantially affect the size of IRL. Eleven snoRNA precursors were detected, with a sequencing coverage higher than 100 reads mapped at each transcript base position. Seven of these snoRNAs were statistically increased in IRL, up to 21-fold for snoZ161_228 and 22-fold for snoR117 (Online Resource 5). The functions of snoRNAs have not been well characterized in plants, but they may act as a central, dynamic, and highly versatile group of small regulatory RNAs, as do miRNAs and snRNAs (DupuisSandoval et al. 2015; Zhu et al. 2016).

\section{Modeling the molecular response}

The black pepper transcripts were clustered by their $A$. thaliana orthologs for modeling the molecular response of the plant to the pathogen. The expression of each ortholog cluster was calculated based on its read counts (Online Resource 6), as described by Nogueira et al. (2016) for non-model transcriptomes. A Z-test was used for each
Fusarium solani f. sp. piperis. The Z-score was obtained using the parametric analysis of gene set enrichment tool of agriGO and indicates significant variation of the GO term categories

AHGC using the read count for each condition (Online Resource 6). A PAGE analysis for the GO terms was performed using AHGC and $\log 2$ of $\mathrm{FC}_{\text {IRL/NIRL }}$ (Fig. 1). GO categories defined as biological processes, cellular processes and molecular functions with significant changes, enrichment or decreases are indicated in Fig. 1. Genes involved in cell-wall organization represented a highly variable category, with a Z-score near 4 . For cellular processes, GO terms clearly decreased for genes associated with plastids and thylakoid lumens (GO:0031978, GO:0009543). In contrast, extracellular regions, especially plant-type cell walls (GO:0009505), were enriched. For molecular functions, many categories were enriched and were associated with antioxidant, transporter, regulatory and catalytic functions. Two subcategories of molecular function, pectinesterase activity (GO:0030599) and carboxylesterase activity (GO:0004091), were highly represented. The global view that was drawn from the analysis of GO enrichment (Fig. 1) indicated high activity in the apoplastic compartment but also in the cell wall, because many genes were involved in dynamic cell-wall remodeling, which likely contributes to the deployment of plant 


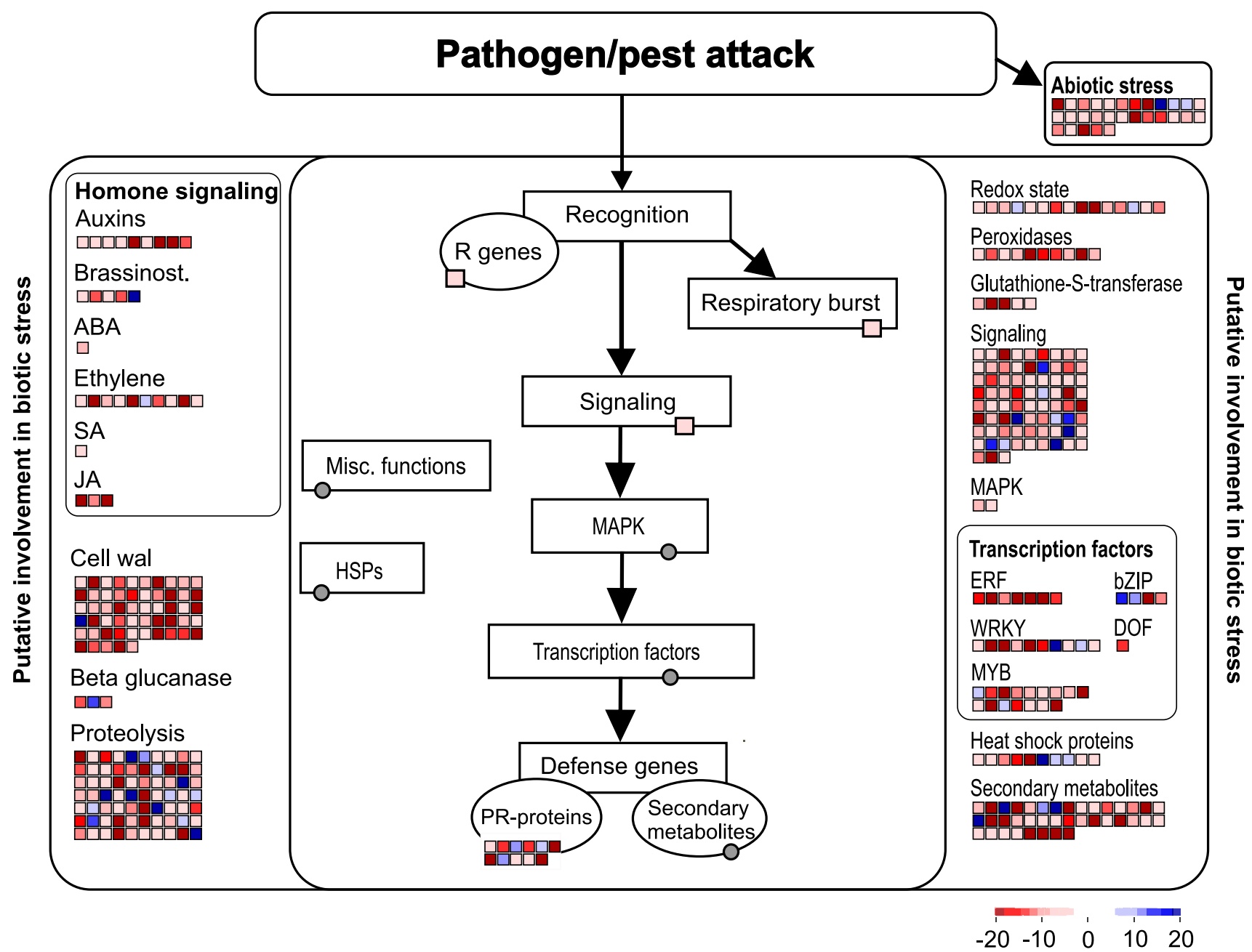

Fig. 2 Biotic-stress category of the MapMan overview showing black pepper transcripts, clustered by Arabidopsis thaliana orthologs, differentially expressed between roots uninfected and infected by Fusarium solani $\mathrm{f}$. sp. piperis. Individual genes are represented by small squares, and their frequencies are indicated by color intensity.

responses against fungal disease (Bellincampi et al. 2014). The presence of plant and not fungal esterase activity was described by Bellincampi et al. (2014) as an ultimate stage of infection, when necrotrophic fungi force plants to collaborate in cell-wall degradation, thus favoring their own metabolism. An important decrease in GO categories for plastids and thylakoids is likely associated with the effect of fungal toxins, such as zearalenone, that can severely damage thylakoids (Chen et al. 2015; Lysøe et al. 2008). These results are in full agreement with our experiment describing plants with or without root-rot symptoms.

Transcripts with significant variation, i.e. $\mathrm{FC}_{\mathrm{IRL} / \mathrm{NIRL}}>5$ and $P<0.001$, were mapped in the biotic-stress category using MapMan to obtain an overview of the black pepper molecular response (Fig. 2). Calculated values for each transcript classified into "biotic-stress related" functional plant category of MapMan are shown in Online Resource
Up- and downregulated genes are shown in red and blue, respectively. Scale bars display the changes in $\mathrm{FC}_{\text {IRL/NIRL. The gray circle indicates }}$ a category without mapped transcripts. The numeric values of each transcript represented in the figure are shown in Online Resource 6

7. Genes associated with biotic stress tended to be upregulated, with three categories highly represented: proteolysis, cell-wall components and secondary metabolism (Fig. 2). An intense proteolysis has been reported for other plant species infected by Fusarium, likely due to the action of the toxins deoxynivalenol and zearalenone (Matthaus et al. 2004; Schwarz et al. 2001).

Most importantly, the data collected in the present study indicated a large contribution of specialized metabolic pathways (or secondary metabolism) in the molecular response. Aspects of such a contribution have been reported (Campos-Bermudez et al. 2013). Many enzymes implicated in the biosynthesis of flavonoids, phenylpropanoids and isoprenoids were upregulated. Key genes controlling the level of isoprenoids through the so-called mevalonate (MVA) and methylerythritol 4-phosphate (MEP) pathways (Hemmerlin et al. 2012), coding for the 
enzymes 3-hydroxy-3-methylglutaryl-CoA reductase $(H M G R)$ and 1-deoxy-D-xylulose-5-phosphate synthase $(D X S)$, were overexpressed 5.1-fold and 8.7-fold ( $\mathrm{FC}_{\mathrm{IRL}}$ / NIRL), respectively. Interestingly, a putative gene encoding geranylgeranyl diphosphate synthase (GGPPS) was upregulated 110-fold in IRL. This PnGGPPS was apparently distant from other known GGPPS. The best hit was the GGPPS sequence of the Chinese peony that shared $34 \%$ aa identity. Many GGPPS have been identified from various species, and their expression has often been associated with plant defensive reactions, as for secologanin biosynthesis during the infection of wheat by Fusarium (Powell et al. 2016). A terpene synthase with $49 \%$ aa identity with a germacrene D synthase from Citrus sinensis was upregulated eightfold in IRL $\left(\mathrm{FC}_{\text {IRL/NIRL }}\right)$, suggesting a role as a defense-specific terpene synthase. A survey of plant-defense genes in our data sets identified many putative disease-resistance proteins: WRKY, bZIP and MYB transcription factors, a guanine nucleotide-binding protein and proteins involved in calcium signaling. The corresponding genes were expressed at levels similar to those observed in other studies of Fusarium-plant interactions, such as the Fusarium-wheat interaction (Powell et al. 2016). Many proteins were associated with drought and abiotic stress, such as ERD (early response to dehydration) and binding immunoglobulin protein. Such modified gene expression would likely account for vessel obstruction and general chlorosis (Carvalho et al. 2014; Rai et al. 2012). Molecular studies of A. thaliana inoculated with Fusarium have identified the hormonal response as an important component of plant defensive responses (Berrocal-Lobo and Molina 2008; Chen et al. 2014). Our results identified a few genes that were up- or downregulated in the abscisic acid and salicylic acid pathways. BAK1 (BRII-associated receptor kinase) was surprisingly downregulated, by 30 -fold for $\mathrm{FC}_{\mathrm{IRL} / \mathrm{NIRL}}$. $B A K 1$ is a central regulator of the brassinosteroid signaling pathway, which could indicate a reduced brassinosteroid response, although brassinosteroids can generally enhance disease tolerance. The $d e-$ etiolated 2 (DET2) and steroid 23-hydroxylase (CPD) genes were upregulated by 5.3-fold and 12.3-fold, respectively. DET2 and CPD are brassinosteroid biosynthetic enzymes. The genes encoding these enzymes were upregulated during biotic and abiotic stress in Cucumis sativus, suggesting a recruitment of the brassinosteroid biosynthetic machinery as part of the defensive response (Xia et al. 2011). These results indicate that the brassinosteroid pathway is involved in plant-pathogen interactions and thus represent a promising target for improving the resistance of black pepper to Fsp infection (Stegmann et al. 2017).

In summary, the transcriptional profiling reported in this study provides valuable information on the nature of the molecular response of black pepper during infection by
Fsp. It has identified previously unknown orphan genes involved in stress response, a strong increase of snoRNA expression, a plant carbohydrate metabolism reprogrammed by Fsp and a major role of isoprenoid biosynthesis and brassinosteroid signalling in black pepper defense. The expression of a gene encoding a terpene synthase that apparently had no functionally characterized counterpart in models such as A. thaliana was an important finding. Further functional characterization of this gene is needed. Our data surprisingly did not implicate pepper alkaloids in the defensive response, which reinforces the role of other metabolic pathways in the interaction with Fusarium. This study provides new insights on black pepper resistance to Fsp, because it addresses the global mobilization of the transcriptome rather than focusing only on specific resistance genes. This analysis also suggests new interesting studies for exhaustively characterizing the black pepper metabolic response for breeding Fsp-tolerant black pepper.

Author contribution statement ECOM, SMCG, SMR, ODdeL, AS, HS, WASJr and IS conceived and designed the study. DGP, EP, HS and SD analyzed and interpreted the data and SD and HS wrote the manuscript.

Acknowledgements The authors thank TR Rommel, G Silva and MSR Barbosa for their technical assistance. SD and HS are grateful to the Centre National de la Recherche Scientifique and Institut National des Sciences Biologiques (INSB) for funding the "Laboratoire International Associé PALMHEAT" CNRS/UFPA.

\section{Compliance with ethical standards}

Conflict of interest The authors of the manuscript have no conflict of interest to declare.

\section{References}

Albuquerque F (1961) Podridão das raízes e do pé da pimenta-doreino

Albuquerque FC, Duarte ML, Benchimol RL, Endo T (2001) Resistência de Piperaceas nativas da Amazônia à infecção causada por Nectria haematococca f. sp. piperis. Acta Amaz $31: 341-348$

Asha S, Nisha J, Soniya EV (2012) In silico characterisation and phylogenetic analysis of two evolutionarily conserved miRNAs (miR166 and miR171) from black pepper (Piper nigrum L.). Plant Mol Biol Rep 31:707-718. doi:10.1007/s11105-012-05325

Balmer D, Mauch-Mani B (2013) More beneath the surface? Root versus shoot antifungal plant defenses. Front Plant Sci 4:256. doi:10.3389/fpls.2013.00256

Bellincampi D, Cervone F, Lionetti V (2014) Plant cell wall dynamics and wall-related susceptibility in plant-pathogen interactions. Front Plant Sci 5:228. doi:10.3389/fpls.2014.00228

Berrocal-Lobo M, Molina A (2008) Arabidopsis defense response against Fusarium oxysporum. Trends Plant Sci 13:145-150. doi:10.1016/j.tplants.2007.12.004 
Bui DC et al (2016) Heat shock protein 90 is required for sexual and asexual development, virulence, and heat shock response in Fusarium graminearum. Sci Rep 6:28154. doi:10.1038/ srep28154

Campos-Bermudez VA, Fauguel CM, Tronconi MA, Casati P, Presello DA, Andreo CS (2013) Transcriptional and metabolic changes associated to the infection by Fusarium verticillioides in maize inbreds with contrasting ear rot resistance. PLoS ONE 8:e61580. doi:10.1371/journal.pone.0061580

Cannesan MA et al (2011) Association between border cell responses and localized root infection by pathogenic Aphanomyces euteiches. Ann Bot 108:459-469. doi:10.1093/aob/mcr177

Carvalho HH et al (2014) The molecular chaperone binding protein $\mathrm{BiP}$ prevents leaf dehydration-induced cellular homeostasis disruption. PLoS ONE 9:e86661. doi:10.1371/journal.pone. 0086661

Chen YC, Wong CL, Muzzi F, Vlaardingerbroek I, Kidd BN, Schenk PM (2014) Root defense analysis against Fusarium oxysporum reveals new regulators to confer resistance. Sci Rep 4:5584. doi:10.1038/srep05584

Chen YE, Cui JM, Su YQ, Yuan S, Yuan M, Zhang HY (2015) Influence of stripe rust infection on the photosynthetic characteristics and antioxidant system of susceptible and resistant wheat cultivars at the adult plant stage. Front Plant Sci 6:779. doi:10.3389/fpls.2015.00779

Conesa A, Gotz S (2008) Blast2GO: a comprehensive suite for functional analysis in plant genomics. Int $\mathrm{J}$ Plant Genomics 2008:619832. doi:10.1155/2008/619832

de Castro GLS, de Lemos OF, Tremacoldi CR, Moraes FKC, dos Santos LRR, Pinheiro HA (2016) Susceptibility of in vitro black pepper plant to the filtrate from a Fusarium solani f. sp. piperis culture. Plant Cell Tissue Organ Cult 127:263-268. doi:10.1007/ s11240-016-1031-4

de Souza CRB, Brígida ABS, Santos RCd, de Nazaré Monteiro Costa C, Darnet SH, Harada ML (2011) Identification of sequences expressed during compatible black pepper-Fusarium solani f. sp. piperis interaction. Acta Physiol Plant 33:2553-2560. doi:10. 1007/s11738-011-0788-7

Du Z, Zhou X, Ling Y, Zhang Z, Su Z (2010) agriGO: a GO analysis toolkit for the agricultural community. Nucleic Acids Res 38:W64-W70. doi:10.1093/nar/gkq310

Duarte MdLR, Albuquerque F (2001) New diseases affecting black pepper crop in Brazil. Int Pepper News Bull 51-57

Dupuis-Sandoval F, Poirier M, Scott MS (2015) The emerging landscape of small nucleolar RNAs in cell biology. Wiley Interdiscip Rev RNA 6:381-397. doi:10.1002/wrna.1284

FAO (2011) Food and Agriculture Organization of the United Nations, statistics of agricultural production. FAO, Rome. http:// www.fao.org/faostat/. Accessed 7 July 2017

Fukutomi M, Hirakata KHM (1981) Studies on the stem rot and root rot diseases of black pepper. Anatomical observations on the distribution on the hiphae of the pathogenic fungus in invading tissues and the blockage of vessel cells. Fitopatol Bras 30(6):586

Gordo SM et al (2012) High-throughput sequencing of black pepper root transcriptome. BMC Plant Biol 12:168. doi:10.1186/14712229-12-168

Gunawardena U, Rodriguez M, Straney D, Romeo JT, VanEtten HD, Hawes MC (2005) Tissue-specific localization of pea root infection by Nectria haematococca. Mechanisms and consequences. Plant Physiol 137:1363-1374. doi:10.1104/pp.104. 056366

Hamada M, Uchida T, Tsuda M (1988) Ascospore dispersion of the causal agent of nectria blight of Piper nigrum. Japanese $\mathrm{J}$ Phytopathol 54:303-308. doi:10.3186/jjphytopath.54.303

Hemmerlin A, Harwood JL, Bach TJ (2012) A raison d'etre for two distinct pathways in the early steps of plant isoprenoid biosynthesis? Prog Lipid Res 51:95-148. doi:10.1016/j.plipres. 2011.12.001

Jaramillo MA, Manos PS (2001) Phylogeny and patterns of floral diversity in the genus Piper (Piperaceae). Am J Bot 88:706-716

Joy N, Abraham Z, Soniya EV (2007) A preliminary assessment of genetic relationships among agronomically important cultivars of black pepper. BMC Genet 8:42. doi:10.1186/1471-2156-8-42

Joy N, Asha S, Mallika V, Soniya EV (2013) De novo transcriptome sequencing reveals a considerable bias in the incidence of simple sequence repeats towards the downstream of 'Pre-miRNAs' of black pepper. PLoS ONE 8:e56694. doi:10.1371/journal.pone. 0056694

Jung K-H, An G (2013) Application of MapMan and RiceNet drives systematic analyses of the early heat stress transcriptome in rice seedlings. J Plant Biol 55:436-449. doi:10.1007/s12374-0120270-0

Kal AJ et al (1999) Dynamics of gene expression revealed by comparison of serial analysis of gene expression transcript profiles from yeast grown on two different carbon sources. Mol Biol Cell 10:1859-1872

Liu Y, Yadev VR, Aggarwal BB, Nair MG (2010) Inhibitory effects of black pepper (Piper nigrum) extracts and compounds on human tumor cell proliferation, cyclooxygenase enzymes, lipid peroxidation and nuclear transcription factor-kappa-B. Nat Prod Commun 5:1253-1257

Lysøe E, Bone KR, Klemsdal SS (2008) Identification of up-regulated genes during zearalenone biosynthesis in Fusarium. Eur J Plant Pathol 122:505-516. doi:10.1007/s10658-008-9318-x

Matthaus K et al (2004) Progression of mycotoxin and nutrient concentrations in wheat after inoculation with Fusarium culmorum. Arch Anim Nutr 58:19-35

Meghwal M, Goswami TK (2013) Piper nigrum and piperine: an update. Phytother Res 27:1121-1130. doi:10.1002/ptr.4972

Menezes IC, Cidade FW, Souza AP, Sampaio IC (2009) Isolation and characterization of microsatellite loci in the black pepper, Piper nigrum L. (piperaceae). Conserv Genet Resour 1:209-212. doi:10.1007/s12686-009-9051-6

Nawrocki EP et al (2015) Rfam 12.0: updates to the RNA families database. Nucleic Acids Res 43:D130-D137. doi:10.1093/nar/ gku1063

Nishimura Y, Kitagishi Y, Yoshida H, Okumura N, Matsuda S (2011) Ethanol extracts of black pepper or turmeric down-regulated SIRT1 protein expression in Daudi culture cells. Mol Med Report 4:727-730. doi:10.3892/mmr.2011.487

Nogueira AF et al (2016) Tetrapod limb and sarcopterygian fin regeneration share a core genetic programme. Nat Commun 7:13364. doi:10.1038/ncomms 13364

Okubara PA, Paulitz TC (2005) Root defense responses to fungal pathogens: a molecular perspective. Plant Soil 274:215-226. doi:10.1007/s11104-004-7328-9

Petre B et al (2012) RNA-Seq of early-infected poplar leaves by the rust pathogen Melampsora larici-populina uncovers PtSultr3;5, a fungal-induced host sulfate transporter. PLoS ONE 7:e44408. doi:10.1371/journal.pone.0044408

Powell JJ et al (2016) The Fusarium crown rot pathogen Fusarium pseudograminearum triggers a suite of transcriptional and metabolic changes in bread wheat (Triticum aestivum L.). Ann Bot. doi:10.1093/aob/mcw207

Rai A, Suprasanna P, D'Souza SF, Kumar V (2012) Membrane topology and predicted RNA-binding function of the "early responsive to dehydration (ERD4) plant protein. PLoS ONE 7:e32658. doi:10.1371/journal.pone.0032658

Ruesch CE et al (2014) The histone H3 lysine 9 methyltransferase DIM-5 modifies chromatin at frequency and represses lightactivated gene expression. G3 (Bethesda) 5:93-101. doi:10. $1534 / \mathrm{g} 3.114 .015446$ 
Schwarz PB, Schwarz JG, Zhou A, Prom LK, Steffenson BJ (2001) Effect of Fusarium graminearum and $F$. Poae infection on barley and malt quality. Monatsschrift Brauwiss 54:55-63

Stegmann $\mathrm{M}$ et al (2017) The receptor kinase FER is a RALFregulated scaffold controlling plant immune signaling. Science 355:287-289. doi:10.1126/science.aal2541

Viegas A, Silva A, Darnet S (2015) HCRA: a hybrid colour-space read-additive method for de novo transcriptomic assembly integrating Illumina and SOLiD datasets. Plant OMICS $8: 581-586$
Xia XJ, Zhou YH, Ding J, Shi K, Asami T, Chen Z, Yu JQ (2011) Induction of systemic stress tolerance by brassinosteroid in Cucumis sativus. New Phytol 191:706-720. doi:10.1111/j.14698137.2011.03745.x

Zhu P, Wang Y, Qin N, Wang F, Wang J, Deng XW, Zhu D (2016) Arabidopsis small nucleolar RNA monitors the efficient prerRNA processing during ribosome biogenesis. Proc Natl Acad Sci USA 113:11967-11972. doi:10.1073/pnas.1614852113 